\title{
An Effective Human Fingerprint Segmentation Method using Watershed Algorithm
}

\author{
Pinaki Pratim Acharjya \\ Assistant Professor, Department of CSE \\ Bengal Institute of Technology and Management \\ Santiniketan, West Bengal, Pin - 731236
}

\author{
Dibyendu Ghoshal \\ Associate Professor, Department of ECE \\ National Institute of Technology \\ Agartala, Tripura, Pin - 799001
}

\begin{abstract}
For more than a century fingerprints ware considered to be the identifying mark for the human beings. Fingerprint is a protected human organ and an effective biometric approach to human or personal identification. It acts like living passwords for humans as its texture is stable throughout the human life. Fingerprints are an impression left by the friction ridges of human finger. This paper contains a very useful image segmentation method for fingerprints segmentation by taking the idea from friction ridges of human finger and also with an effective storage capacity for the segmented images. Watershed algorithm depends on ridges to perform a proper segmentation, a property that is often fulfilled in contour detection where the boundaries of the objects are expressed as ridges. The tool we have used is MAT LAB, typically using the MAT LAB editor.
\end{abstract}

\section{Keywords}

Image segmentation, Fingerprints, Watershed Algorithm.

\section{INTRODUCTION}

Image segmentation plays a significant role in image processing. Several methods have been proposed in the last few decades [1-2] but still it is a great problem for automatic image segmentation [3-5] which is challenging the engineers. Image segmentation is a process of dividing images into regions depending on the color and object characteristics that is present in the image. The main goal behind the concept of image segmentation is to achieve better images then the original foe easier analyzing, understanding and which should be more meaningful. The segmented result must be correct for analysis, prediction and diagnosis. In recent days watershed algorithm [6], [19] is becoming a popular image segmentation method for several applications in various fields [7-12].

In present study this watershed algorithm has been used for segmentation of human fingerprint impressions [13-14]. Fingerprint analysis is a very important issue and its applications [15-16] are mostly for identification of humans. Fingerprints offer an infallible means of personal identification. In 1686, Marcello Malpighi, a professor of anatomy at the University of Bologna, noted in his treatise; ridges, spirals and loops in fingerprints. He made no mention of their value as a tool for individual identification. For the last century fingerprints ware considered as a unique identity for human beings. But before that it was not so important, because it was only considered as a password or identification mark for humans in the late 19th century that all fingerprints are different from one another. In recent days fingerprints are the very basis for criminal history foundation at every police agency on earth for accurate identification of criminals as forensic evidence in all over the world. Every person has a different set of fingerprints which is a visible pattern formed on the tip of a finger. For several reasons we need to store these fingerprints in a database and among them one of the main reason is they are used for analysis of forensic evidence worldwide. For storing several fingerprint impressions a huge database is needed, where the size of the database is also a matter of consideration. A huge database needs a huge amount of memory space. If we can reduce the size of the data then we can store more no of data in the same memory space. This paper proposes an effective segmentation method using watershed algorithm [17-20] for segmentation of fingerprint impressions and memory space reduction.

For better understanding and explanation the division of this paper as follows. The section 2 describes the basic theories related to fingerprints and watershed algorithm. In section 3 , the proposed method is discussed. In section 4, implementation results and discussions are discussed with the effectiveness of the proposed method. Finally conclusion and references are given in section 5 .

\section{BASIC THEORY}

\subsection{FINGERPRINTS}

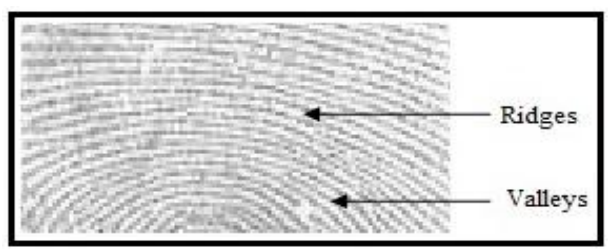

Fig. 1: Ridges and Valleys in finger print.

If we take a microscopic picture of a fingerprint we will find some single curve segment. Several curve segments combines together and forms a fingerprint pattern. The curve segments that flow in various patterns across fingerprints are called ridges and the spaces between ridges are valleys. Figure 1 will give us a clearer idea.

For verification and matching of human fingerprints we need to concern on several features of fingerprint pattern. For employing of some imaging technology, it is also very important to know the basic structure and characteristics of human pingerprints. There is mainly three basic patterns, arch, loop and whorl. The arch ridges enter from one side of the finger, rise in the center forming an arc, and then exit the other side of the finger. The loop ridges enter from one side of a finger, form a curve, and then exit on that same side. And the whorl Ridges form circularly around a central point on the finger. Figure 2 illustrates the three basic patters for classification of human fingerprints. 


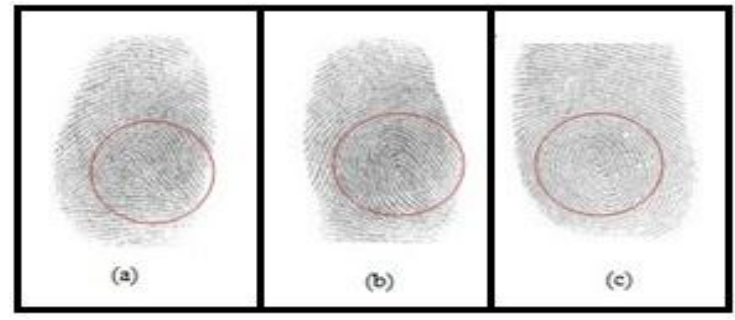

Fig. 2: Different fingerprint patterns: (a) arch, (b) loop, and (c) whorl.

\subsection{Watershed Algorithm}

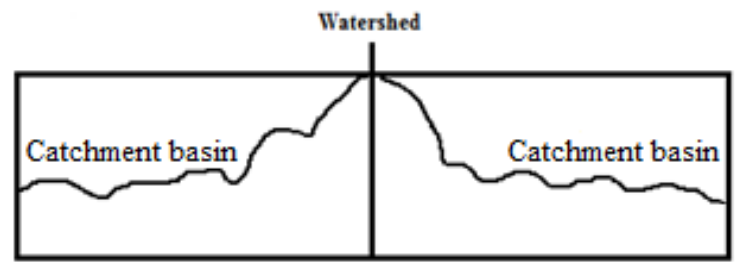

Fig.3: Watershed segmentation-local minima yield catchment basins; local maxima define the watershed lines.

The idea behind watersheds has been introduced by $\mathrm{S}$. Beucher and C. Lantuéjoul in 1979. A gray scale image can be interpreted as the topographic image of landscape. Using the features of these images, the technique of digital image processing called Watershed Transform. It consists in placing a water source in each regional minimum, to flood the relief from sources, and build barriers when different sources are meeting. The resulting set of barriers constitutes a watershed by flooding.

\subsection{Mathematical Formulae of Watershed Algorithm}

Assume, $M_{i}$ where $i=1$ to $n$ be the set of coordinates points in the regional minima of the image $\mathrm{P}(\mathrm{x}, \mathrm{y})$ and $\mathrm{C}\left(\mathrm{M}_{\mathrm{i}}\right)$ be the coordinates points of catchment basins associated with the regional minima $\mathrm{M}_{\mathrm{i}}$

$\mathrm{T}_{\mathrm{n}}=\{(\mathrm{s}, \mathrm{t}) \mid \mathrm{P}(\mathrm{s}, \mathrm{t})<\mathrm{n}\}$

(1)

Where,

$\mathrm{T}[\mathrm{n}]=$ set of points in $\mathrm{P}(\mathrm{x}, \mathrm{y})$ which are lying below the plane $\mathrm{p}(\mathrm{x}, \mathrm{y})=\mathrm{n}$

$\min , \max =$ minimum or maximum gray level value.

$\mathrm{n}=$ stage of flooding varies from $\min +1$ to $\max +1$

Let $C_{n}\left(M_{1}\right)$ be the set of points in the catchment basin associated with $M_{1}$ that are flooded at stage $n$.

$\mathrm{C}_{\mathrm{n}}\left(\mathrm{M}_{1}\right)=\cap\left\{\mathrm{C}\left(\mathrm{M}_{1}\right), \mathrm{T}[\mathrm{n}]\right\}$

(2)

Where,

$$
\mathrm{Cn}\left(\mathrm{M}_{\mathrm{i}}\right)=\left\{\begin{array}{l}
\mathbb{1}_{1} \text { if }(\mathrm{x}, \mathrm{y}) \in \mathrm{C}\left(\mathrm{M}_{\mathrm{i}}\right) \operatorname{and}(\mathrm{x}, \mathrm{y}) \in \mathbb{T}[\mathrm{n}] \\
0, \text { otherwise }
\end{array}\right.
$$

$\mathrm{C}[\mathrm{n}]$ be the union of flooded catchment basin portions at the stage $n$.

Where,

$\mathrm{C}[\mathrm{n}]=\mathrm{C}_{\mathrm{n}}\left(\mathrm{m}_{1}\right) \cup \mathrm{C}_{\mathrm{n}}\left(\mathrm{m}_{2}\right) \ldots \ldots . \mathrm{C}_{\mathrm{n}}\left(\mathrm{m}_{\mathrm{R}}\right)$

(4)

$\mathrm{C}[\max +1]=\mathrm{C}\left(\mathrm{m}_{1}\right) \cup \mathrm{C}\left(\mathrm{m}_{2}\right) \ldots \ldots . \mathrm{C}\left(\mathrm{m}_{\mathrm{R}}\right)$

(5)

If the algorithm keeps on increasing flooding level then $\mathrm{C}_{\mathrm{n}}\left(\mathrm{M}_{\mathrm{i}}\right)$ and $\mathrm{T}[\mathrm{n}]$ will either remain constant or increase. Algorithm initializes $\mathrm{C}[\mathrm{min}+1]=\mathrm{T}[\mathrm{min}+1]$, and then proceeds recursively by assuming that at step $n C[n-1]$ has been constructed.

Let, $\mathrm{G}$ be set of connected components in $\mathrm{T}[\mathrm{n}]$ and for each connected component $\mathrm{g} \in \mathrm{G}[\mathrm{n}]$, there possibilities will arise

- $\mathrm{g} \cap \mathrm{C}[\mathrm{n}-1]$ is empty.

- $\quad g \cap C[n-1]$ contains one connected component of $\mathrm{C}[\mathrm{n}-1]$.

- $\quad g \cap C[n-1]$ contains more than one connected component of $\mathrm{C}[\mathrm{n}-1]$

\section{PROPOSED APPROACH}

This approach deals with catchment basins and watershed ridge lines in an image by assuming it as a surface where light pixels are low. In every fingerprint some curve segments or ridges are there and several curve segments or ridges combines together and forms a fingerprint pattern. In our proposed approach we have tried to deal with these ridges by applying watershed algorithm.

First insert the image into MAT LAB editor using 'imread' function. The 'imread' function in MAT LAB reads an image which may be grayscale or color image from the specified file. If the file is not found in the current folder, or in a folder on the MATLAB path, we have to specify the full pathname. This function supports several formats like, Windows bitmaps (always supported), JPEG, JPEG 2000 files, Portable Network Graphics, Portable image format, TIFF files and many more formats. In the next step the color images have been converted into gray level images using 'rgb2gray' function. If the input is a color or rgb image of class uint8, uint16, single, or double, the resultant image will be of the same class as the input image. Watershed algorithm works only on gray scale image because a grey scale image may be considered as a topographic relief. After converting the image into gray scale we compute the complement of the gray scale image using MAT LAB function 'imcomplement'. In the complement of a binary image, zeros become ones and ones become zeros and as a result, in the output image, bright and dark regions being reversed. Next we compute the H-minima transform using 'imhmin' function. This function is the algorithemic basis for morphological reconstruction in image processing toolbox. And finally we compute the watershed regions as a segmented image using 'watershed' function. The detailed algorithm is as the following: 


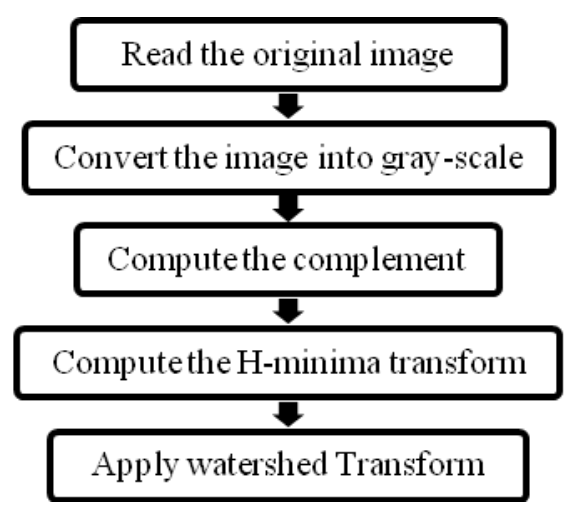

\section{IMPLEMENTATION RESULTS AND DISCUSSION}

The results obtained by the proposed work are shown below in figure 4 to 8 . The proposed approach had been implemented on different fingers of one of the authors, where the segmented results are very clear. In following figures, (a), (b), (c), (d) and (e) are the step wise resultant images of the proposed approach where, (a) is the original image, (b) is the gray scale image of the original one, (c) is the complement of the gray scale image, (d) is the H-minima transformed image and finally we gets the segmented image (e) by applying watershed algorithm.

In Table 1, the entropy for original images with their segmented results has been measured. The performance of Segmentation algorithm can be measured with the help of Entropy and as in term of visual quality of the original image and the resulted image. The image entropy can provide a good level of information to describe a given image. Low entropy images, such as those containing a lot of black sky, have very little contrast and large runs of pixels with the same values. An image that is perfectly flat will have entropy of zero. Consequently, they can be compressed to a relatively small size. On the other hand, high entropy images such as an image of heavily cratered areas on the moon have a great deal of contrast from one pixel to the next and consequently cannot be compressed as much as low entropy images.

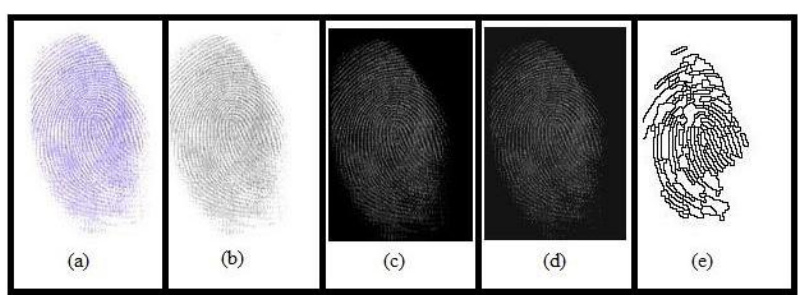

Fig. 4: Example of our approach with little finger.

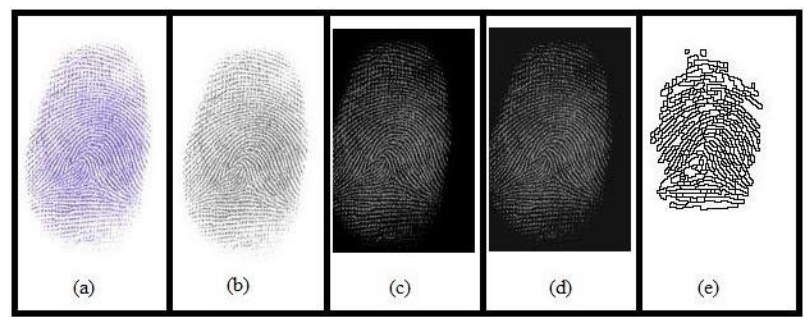

Fig. 5: Example of our approach with ring finger.

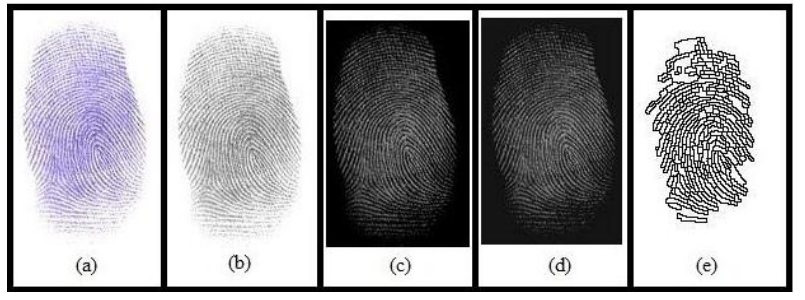

Fig. 6: Example of our approach with middle finger.

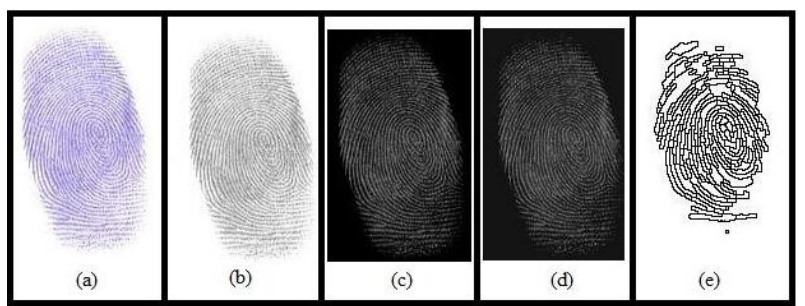

Fig. 7: Example of our approach with grooming finger.

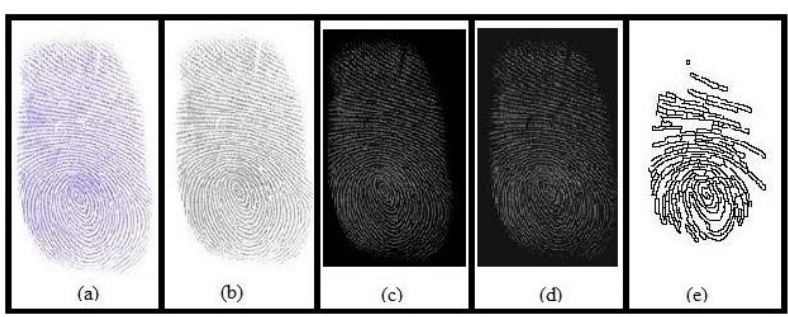

Fig. 8: Example of our approach with thumb finger.

Table 1: statistical measurement

\begin{tabular}{|c|c|c|}
\hline Name of fingers & $\begin{array}{c}\text { Entropy of } \\
\text { original image }\end{array}$ & $\begin{array}{c}\text { Entropy of } \\
\text { segmented image }\end{array}$ \\
\hline Little finger & 5.4766 & 1.0281 \\
\hline Ring finger & 5.5974 & 0.9537 \\
\hline Middle finger & 5.6865 & 0.9820 \\
\hline Grooming finger & 5.2182 & 0.9620 \\
\hline Thumb finger & 5.4733 & 0.9969 \\
\hline
\end{tabular}

\section{CONCLUSIONS}

The present study has aimed at to provide an easy method of the segmentation of the human fingerprints based on mathematical morphology. The concept of watershed algorithm has been used for segmentation purpose. The present method has been found to yield better output in term of image clarity and sharpness with the avoidance of over segmentation. In practical applications, fingerprints are unique feature for identification and verification of humans, and as well as we need to maintain several databases for storing the images of fingerprints and the sizes of the databases are a major concerned issue. We can store the segmented images of fingerprints instead of the original images to reduce the size of 
the databases. The final result of segmentation depends upon the quality of scanner and the inkpad which we use.

\section{ACKNOWLEDGMENT}

The authors acknowledge the constant inspiration and encouragement from Professor P. K. Bose, Director, National Institute of Technology, Agartala.

\section{DEDICATION}

One of the others (Dibyendu Ghoshal) dedicates the entire study to the loveliest and loving memory of his only one and younger sister Kumari Sumita Ghoshal who herself was a gem of the scholars, a symbol of wisdom and art, peerless beauty and simplicity, unfathomable knowledge and generosity.

\section{REFERENCES}

[1] C. Gonzalez, Richard E. Woods, Digital Image Processing, Addison westly pub.company.

[2] C.Gonzalez, Richard E.Woods, Digital Image Processing , Addison westly pub.company.

[3] L. Vincent and P. Soille, "Watersheds in digital spaces: an efficient algorithm based on immersion simulations," IEEE Trans. Pattern Anal. Mach. Intell., vol. 13, no. 6, pp. 583-598, Jun. 1991.

[4] S. Beucher, "Watersheds of functions and picture segmentation," in Proc. IEEEInt. Conf. Acoustic, Speech, Signal Processing, pp. 1982-`931, 1982.

[5] Gonzalez \& Woods, Digital Image Processing, 3rd edition, Prentice Hall India, 2008.

[6] P.Jackway, "Gradient watersheds in morphological scale space," IEEE Trans. Image Processing vol. 15, pp. 913921, June, 1996.

[7] S. Beucher, "Watershed, hierarchical segmentation and water fall algorithm," in Mathematical Morphology and Its Applications to Image Processing, Dordrecht, The Netherlands: Kluwer, 1994, pp. 69-76.

[8] E. N. Mortrnsen and W. A. Barrett, "Toboggan-based intelligent scissors with a four-paramreer edge model," in Proc. IEEE Conf. Computer Vision and Pattern Recognition, pp. 452-458, 1999.
[9] Vicent L. Solille P, Watershed in digital spaces, "An efficient algorithm based immersion simulations," IEEE Transections PAMI, pp. 538-598, 1991.

[10] M. Couprie and G. Bertrand, "Topological grayscale watershed transformation," in Proc. SPIE Vision Geometry V, vol. 3168, pp. 136-146, 1997.

[11] C. Riddell, p. Brigger, R. E. Carson and S. L. Bacharach, "The watershed algorithm: a method to segment noisy PET transmission images," IEEE Trans. Nucl. Sci., vol. 46, no. 3, pp. 713-719, Mar, 1999.

[12] M. W. Hansen and W. E. Higgins, "Watershed-based maximum-homogeneity filtering," IEEE Trans. Image Process., vol. 8, no. 7, pp. 982-988, jul. 1999.

[13] U. Halici, Turkey,L.C. Jain, “ Introduction to finger print Recognition,” Australia \& A.Erol, Turkey.

[14] J. Berry, "The history and development of fingerprinting," in Advances in Fingerprint Technology, (H. C. Lee and R. E. Gaensslen, ed.s), CRC Press, Florida, 1994, pp. 1-38, 1994.

[15] W. S. Chen and C. L. Kuo, "Apparatus for Imaging Fingerprint or Topgraphic Relief Pattern on the Surface of an Object," US Patent 5448649, 1995.

[16] A. K. Jain, L. Hong, S. Pankanti, and R. Bolle, "An Identity-Authentication System Using Fingerprints," Proceedings of the IEEE, Vol. 85, No. 9, pp. 1365-1388, 1997.

[17] K. Haris,"Hybrid image segmentation using watersheds and fast region merging," IEEE Trans Image Processing, 7(12), pp. 1684-1699, 1998.

[18] F. Meyer, S. Beucher, "Morphological Segmentation," Journal of Visual Communication and Image Representation, 1, pp. 21-46, 1990.

[19] D. Wang, "Unsupervised video segmentation based on waterseds and temporal traking," IEEE Trans. Circuits Syst. VideoTechnol., vol. 8, no. 5, pp. 539-546, May 1998.

[20] Rafael C. Gonzalez, Richard E. Woods, Steven L. Eddins, "Digital Image Processing Using MATLAB," Second Edition, Gatesmark Publishing, 2009 